REVIEW ARTICLE

\title{
High unbound bilirubin for age: a neurotoxin with major effects on the developing brain
}

\author{
Rowena Cayabyab ${ }^{1}$ and Rangasamy Ramanathan ${ }^{1}$
}

Neonatal hyperbilirubinemia is one of the most frequent diagnoses made in neonates. A high level of unconjugated bilirubin that is unbound to albumin is neurotoxic when the level exceeds age-specific thresholds or at lower levels in neonates with neurotoxic risk factors. Lower range of unbound bilirubin results in apoptosis, while moderate-to-high levels result in neuronal necrosis. Basal ganglia and various brain stem nuclei are more susceptible to bilirubin toxicity. Proposed mechanisms of bilirubin-induced neurotoxicity include excessive release of glutamate, mitochondrial energy failure, release of proinflammatory cytokines, and increased intracellular calcium concentration. These mechanisms are similar to the events that occur following hypoxic-ischemic insult in neonates. Severe hyperbilirubinemia in term neonates has been shown to be associated with increased risk for autism spectrum disorders. The neuropathological finding of bilirubin-induced neurotoxicity also includes cerebellar injury with a decreased number of Purkinje cells, and disruption of multisensory feedback loop between cerebellum and cortical neurons which may explain the clinical characteristics of autism spectrum disorders. Severe hyperbilirubinemia occurs more frequently in infants from low- and middle-income countries (LMIC). Simple devices to measure bilirubin, and timely treatment are essential to reduce neurotoxicity, and improve outcomes for thousands of neonates around the world.

Pediatric Research (2019) 85:183-190; https://doi.org/10.1038/s41390-018-0224-4

\section{INTRODUCTION}

Neonatal jaundice is a very common condition occurring in $60-80 \%$ of all healthy term and late preterm neonates. It is the most common clinical diagnosis in neonates due to elevated unconjugated bilirubin (UCB). In the majority of infants, jaundice resolves spontaneously and causes no harm; however, in some babies, significant hyperbilirubinemia can develop. Among late preterm and term infants, total serum bilirubin (TSB) $>95$ th percentile occurs in 81/1000 live births and TSB >99.9th percentile occurs in $1 / 1000$ live births. ${ }^{1}$ When treated with phototherapy or exchange transfusion, TSB levels $>25 \mathrm{mg} / \mathrm{dL}$ (above 95th percentile) were not associated with adverse neurodevelopmental outcomes in infants born at or near term. ${ }^{2}$ Among infants with severe hyperbilirubinemia who are untreated or inadequately treated with phototherapy or exchange transfusion, due to lack of resources or lack of knowledge about bilirubin-induced neurotoxicity, the incidence of brain injury is between 0.14 and $0.16 \%{ }^{3}$ Lipophilic UCB is neurotoxic and can cause injury to the basal ganglia and various brain stem nuclei, resulting in acute bilirubin encephalopathy $(A B E)$ and may progress to chronic bilirubin encephalopathy (CBE) or kernicterus. Hyperbilirubinemia with signs of encephalopathy should be considered a neurologic emergency and treated immediately because the outcome is related in part to the duration of exposure to excessive free UCB. ${ }^{4}$ The risk for kernicterus is about 1 in 16 when TSB is $>25 \mathrm{mg} / \mathrm{dL}$ and 1 in 5.5 when TSB is $>30 \mathrm{mg} / \mathrm{dL}$. Reported incidence of CBE in Canada is 1 in 44,000 live births, ${ }^{5} 0.9 / 100,000$ live births in the United Kingdom, ${ }^{6}$ and 0.6 per 100,000 live births in Denmark. ${ }^{7}$ However, in low- and middle-income countries (LMIC), especially, Sub-Saharan Africa and South East Asia, kernicterus-related disorders (KSD) are significantly underreported. The incidence of severe neonatal jaundice ranges between 4 and $49 \%$ and the incidence of $A B E$ ranges from 0.5 to $30 \%$. Furthermore, neonates with glucose-6-phosphate dehydrogenase (G6PD) deficiency are also disproportionately overrepresented in these parts of the world. ${ }^{8}$ In term infants, regardless of the rapidity of intervention or clinical assessment of the severity of acute bilirubin toxicity, either before or after treatment have been observed to develop some degree of irreversible neurological sequelae. ${ }^{9,10}$

Regional brain development and vulnerability determine periods during which moderate hyperbilirubinemia could result in auditory versus motor predominant symptoms of bilirubininduced neurologic dysfunction (BIND). These manifestations are distinct from those associated with exposure to high levels of UCB that is known to cause severe neonatal motor symptoms and long-term sequelae such as dyskinetic cerebral palsy (CP). ${ }^{11}$ BIND refers to a wider spectrum of neurologic manifestations. In these disorders, aside from $A B E$ and kernicterus, it also includes less severe forms of auditory neuropathy. ${ }^{10}$ The prevalence of BIND is higher in children with a neonatal history of severe hyperbilirubinemia, and in the presence of hemolysis, asphyxia, or sepsis. $8,12,13$

The implementation of universal bilirubin screening using transcutaneous bilirubin (TCB) or total serum bilirubin (TSB) measurements, ${ }^{14-16}$ has led to increased identification of newborns with severe jaundice and timely initiation of phototherapy. ${ }^{17}$ However, the effects of universal screening on the rates of bilirubin encephalopathy are unknown. ${ }^{18}$ Despite the efforts to eliminate permanent and irreversible brain damage due to bilirubin encephalopathy, and kernicterus, these conditions

\footnotetext{
${ }^{1}$ Keck School of Medicine of University of Southern California, Division of Neonatology, Department of Pediatrics, LAC+USC Medical Center, Los Angeles, CA, USA
} Correspondence: Rangasamy Ramanathan (ramanath@usc.edu)

Received: 9 May 2018 Revised: 15 October 2018 Accepted: 17 October 2018

Published online: 5 December 2018 
continue to occur in the third millennium in the western world, but most importantly in LMIC. $^{19}$

\section{BILIRUBIN PRODUCTION}

Bilirubin is a yellow pigment derived from heme catabolism in the reticuloendothelial system and exists in the blood mostly bound to albumin (Fig. 1). Increased production of bilirubin is due to increased breakdown of erythrocytes containing fetal $\mathrm{Hgb}$, ineffective erythropoiesis, along with decreased conjugation due to immaturity of liver enzymes, as well as a unique neonatal phenomenon of enterohepatic recirculation of bilirubin during the first week of life. ${ }^{20,21}$ Bilirubin production rate is between 6 and 10 $\mathrm{mg} / \mathrm{kg} /$ day, which is two to three times higher than in adults. The possible beneficial role of bilirubin as the most important antioxidant in the human system, coupled with the presence of multiple mechanisms to preserve and increase bilirubin production has led to speculate that there may be an as-yetunrecognized beneficial role for bilirubin as an antioxidant in newborn infants, especially around the time of birth, which is a major period of oxidant challenge.

This transitional imbalance of production and elimination is normal but can be upset in several conditions, which are often associated with increased bilirubin production and increased resorption of bilirubin. All these factors may further increase an infant's bilirubin load to cause severe hyperbilirubinema, especially in infants with hemolysis, asphyxia, and sepsis, and among those with genetic predisposition. ${ }^{12,16,22}$ With the use of wholegenome sequencing, 32 gene mutations have been identified to date, causing hyperbilirubinemia of unknown etiology (personal communication, Fulgent Genetics).

UCB is bound to albumin in the systemic circulation before conjugation in the hepatocyte where it combines enzymatically with glucuronic acid to form bilirubin monoglucuronide and diglucuronides and excreted into the bile and the gut. In the newborn, much of the conjugated bilirubin in the intestine is hydrolyzed back to UCB by the enzyme, $\beta$-glucuronidase and is reabsorbed into the bloodstream by way of enterohepatic circulation, an important contributor to neonatal jaundice. ${ }^{12,23,24}$ Breast milk jaundice is likely due to increased enterohepatic circulation, secondary to $\beta$-glucuronidase activity in breast milk.

\section{MECHANISMS OF BILIRUBIN NEUROTOXICITY}

The fraction of unbound bilirubin, also known as free bilirubin or non-albumin-bound UCB increases significantly as the TSB approaches the binding capacity of albumin $(1 \mathrm{~g}$ of albumin binds to $8 \mathrm{mg}$ of bilirubin, $\sim 1: 1$ on a molar basis) Important determinants of neurologic injury by bilirubin are concentration

Simplified metabolic pathway of Bilirubin formation

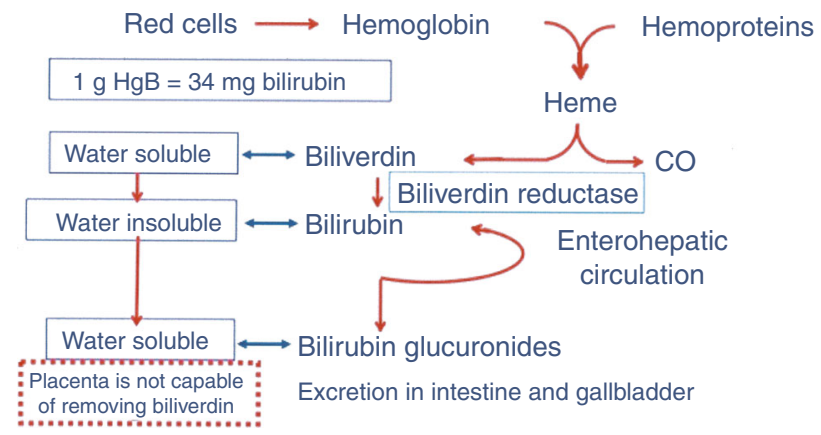

Fig. 1 Bilirubin production

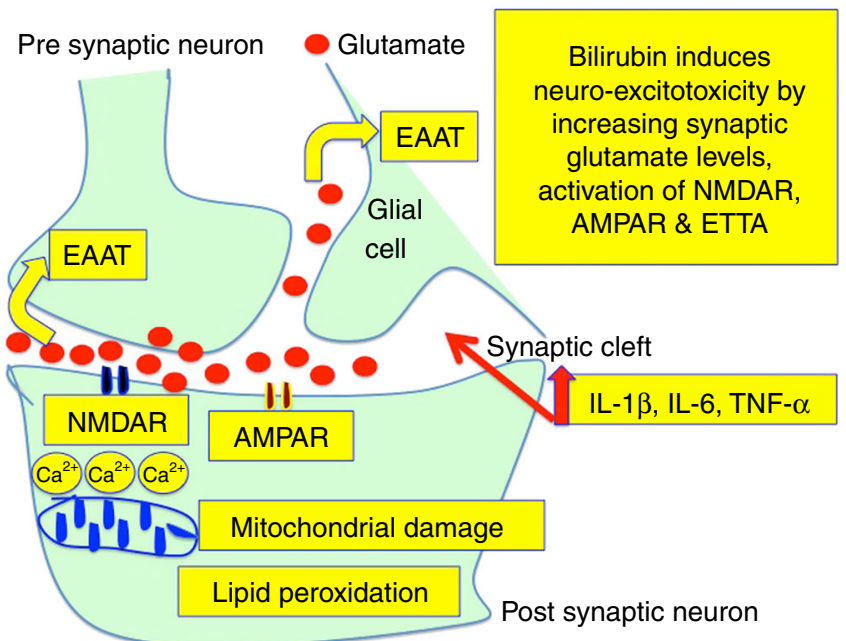

Fig. 2 Proposed mechanisms for bilirubin-induced neurotoxicity

of unbound $\mathrm{UCB}$, concentration of serum albumin, bilirubin binding by albumin, concentration of hydrogen ions, blood-brain barrier permeability, neuronal susceptibility and central nervous system immaturity secondary to prematurity, ${ }^{25,26}$ as well as the duration and degree of hyperbilirubinemia. ${ }^{27,28}$ Depending on these associated risk factors, neurotoxic effects may be transient or permanent. ${ }^{21}$ Free UCB which is lipid soluble crosses the blood-brain barrier and affects the plasma membranes and mitochondria of the neurons, microglial cells, and astrocytes, resulting in ${ }^{29,30}$ microglial and astrocyte activation, impaired myelination, and neuronal death. ${ }^{31,32}$

Proposed mechanisms of bilirubin-induced neurotoxicity include excessive release of glutamate, mitochondrial energy failure, release of proinflammatory cytokines (Fig. 2), and increased intracellular calcium concentration. ${ }^{33}$ Consistent with neuronal injury are the perturbations in membrane permeability and function. Impairment of glutamate transport in astrocytes and neurons causes an increase in extracellular glutamate, resulting in excessive activation of neuronal $\mathrm{N}$-methyl D-aspartate (NMDA) receptors with an increase in cytosolic calcium, generation of free radicals, and neuronal necrosis or apoptosis. ${ }^{32,34-36}$ Astrocytes also release cytokines such as tumor necrosis factor alpha (TNF-a) and interleukin $1 \beta$ (IL-1 $\beta$ ), impairing glutamate transport leading to the accumulation of extracellular glutamate. ${ }^{36}$ Effect on mitochondria is damage of oxidative phosphorylation resulting in diminished energy production, increased mitochondrial permeability, and release of cytochrome $C$ into the cytosol that can lead to apoptotic neuronal death by binding to apoptosis proteaseactivating factor 1 , and activation of caspases. ${ }^{37}$ The final common pathway for both the plasma membrane effects with excitotoxicity and the mitochondrial disturbances includes an increase in cytosolic calcium and generation of free radicals, resulting in apoptotic or necrotic cell death or both. ${ }^{38-40}$ In a review article published in 2006, Watchko concluded that there is strong evidence that bilirubin-induced injury is first and foremost membranous in origin and the neurotoxic cascade is initiated at the plasma, mitochondrial, and/or endoplasmic reticulum membrane level. ${ }^{39}$ These membrane effects, in turn, may lead to the genesis of neuronal excitotoxicity, mitochondrial energy failure, or increased intracellular calcium concentration $\left[\mathrm{Ca}^{2+}\right]$.

Bilirubin is also toxic to oligodendrocytes through the generation of reactive oxygen species. ${ }^{41}$ Myelination is altered in preterm infants with kernicterus as recent evidence has shown that bilirubin is toxic to both myelinated and unmyelinated axons. In vitro, UCB causes decreased oligodendroglia differentiation and reduction in the number of myelinating oligodendroglia. ${ }^{29,31}$ 
Another possible mechanism in bilirubin neurotoxicity is through inhibition of phosphorylation of several proteins catalyzed by a number of protein kinases. Protein phosphorylation plays a key role in cellular regulation. ${ }^{42}$

Animal data suggest that the level of UCB in the brain determines whether and to what extent bilirubin neurotoxicity occurs. ${ }^{39,40}$ The results of human neonatal studies have suggested that the neurologic outcome of hyperbilirubinemia correlates better with serum or plasma free bilirubin than TSB. TSB measures only bilirubin bound to albumin. There is currently no clinically available sensitive and accurate method for measurement of free UCB. ${ }^{43-46}$ Recent studies have shown that unbound bilirubin is a more sensitive and specific predictor of auditory neuropathy spectrum disorder than TSB. ${ }^{47,48}$

\section{DISTRIBUTION OF CENTRAL NERVOUS SYSTEM INJURY}

Bilirubin has a certain topographical distribution and it is unclear why bilirubin-induced neurotoxicity is so regional and neuronal cell specific. The most injurious form of $\mathrm{CBE}$, also known as kernicterus involves the basal ganglia, particularly, globus pallidus, substantia nigra, reticulata, subthalamic nuclei, brain stem, auditory, vestibular, and oculomotor nuclei, hippocampus, and cerebellum. ${ }^{27}$ In most cases, globus pallidus, external and internal subthalamic nuclei have shown moderate and marked loss of neurons, demyelination, and prominent gliosis. ${ }^{49-51}$ Hypothesis for the region-specific nature of bilirubin-induced neurotoxicity may reflect differences in cerebral regional blood flow, bilirubin uptake, tissue binding and clearance, or to differential sensitivity of neurons to injury. ${ }^{27,39,52}$ Subtle neurodevelopmental disabilities may be related to more uniform distribution of bilirubin in the brain parenchyma, although it may include disturbances of sensory and sensorimotor integration, central auditory processing, coordination, and control of muscle tone..$^{53,54}$

\section{BILIRUBIN AND CEREBELLAR INJURY}

Bilirubin-induced neurotoxicity is characterized by cerebellar injury with a decreased number of Purkinje cells. ${ }^{50}$ In a mouse model with null mutation in the uridine diphosphate glucuronosyl transferase (Ugt1) gene resulting in hyperbilirubinemia, characteristic neuropathological findings were cerebellar neurodegeneration, cerebellar hypoplasia, and neuronal cell death. Furthermore, these mutant mice also showed several of the clinical and histopathological characteristics of BIND, including severe motor impairment, slow movement, decreased feeding, and major changes in cerebellar architecture. ${ }^{55}$

\section{TIMING OF PERINATAL INSULT}

The timing of perinatal bilirubin insult may be important in determining the pattern of brain injury and the extent of neurological dysfunction after birth. Studies have shown that there are windows of developmental susceptibility of the central nervous system (CNS) to bilirubin toxicity influencing the location of the neuropathological damage. ${ }^{29}$ In vitro studies have shown that bilirubin cytotoxicity causing apoptosis is enhanced in undifferentiated astrocytes and neurons, as compared with more mature cells. ${ }^{56}$ Alterations in neuronal/axonal units involving a decreased number of axonal branches, smaller growth cone area, and reduced dendritic spines and synapses were observed in immature neurons exposed to UCB, while only subtle damages were produced in postnatal cerebellar development with later exposures. ${ }^{57}$ These findings suggest an acquired resistance to bilirubin neurotoxicity with increasing postnatal age and that age at exposure determines the pattern of bilirubin-induced CNS toxicity probably due to developmentally regulated maturation of the different brain regions. ${ }^{58}$ In infants $>34$ weeks of gestation, the motor predominant type of kernicterus usually develops, while auditory predominant type of kernicterus occurs in infants born at an earlier gestational age. This may be secondary to earlier, faster myelination progression in sensory pathways (somatosensory, vision, and auditory) compared to motor pathways. ${ }^{29}$

\section{RISK FACTORS THAT INCREASE NEUROTOXICITY}

Low serum albumin concentrations $(1.4-2.1 \mathrm{mg} / \mathrm{dL})$ with a reduced bilirubin binding capacity in the serum are frequently observed in cases of kernicterus with low bilirubin levels. ${ }^{59}$ In addition, substances such as free fatty acids, and drugs, like sulfisoxazole preferentially bind to albumin at its high-affinity sites, while aromatic anions displace bilirubin from the secondary low-affinity sites. ${ }^{60}$ This finding of the decrease in bilirubin binding affinity was associated with a lower peak UCB in infants $\leq 30$ weeks of gestation. ${ }^{61}$

Animal studies have shown that infection with $E$. coli endotoxin increased TSB levels and subsequent accumulation in the brain. ${ }^{62}$ Sepsis-related systemic inflammatory response may increase the permeability of the blood-brain barrier and enhance bilirubin entrance into the CNS.63 Fetal inflammatory response syndrome can lead to CNS injury via direct cytokine effects, making the brain more vulnerable to bilirubin-induced CNS injury even at low TSB levels generally not thought to pose a neurotoxic risk. ${ }^{64}$ Significant increase in neurologic abnormalities was observed at a peak TSB of $12-13 \mathrm{mg} / \mathrm{dL}$ that was enhanced as placental inflammation became more severe and was present only in preterm infants born before 34 weeks of gestation. There is also a link between cerebral hypoxia and bilirubin, where kernicterus can occur in the presence of low bilirubin with preferential bilirubin accumulation in cerebral areas severely injured as a result of perinatal asphyxia. These findings suggest that bilirubin may enhance hypoxic brain damage. ${ }^{64}$

The homozygous (jj) Gunn rat animal (absent UGT1A1 enzyme) has a peak serum bilirubin and UCB peaks at postnatal day 16 and remains elevated throughout the animal's life. The jj Gunn rat exhibits many of the clinical signs and histopathologic lesions that occur in humans suffering from kernicterus. These rats manifest a wide spectrum of neurologic involvement ranging from near absence of clinical symptoms to the presence of severe abnormalities that include opisthotonus, seizures, and death. Cell loss and gliosis are most prominent in the auditory nuclei of the brain stem, oculomotor nuclei, cerebellum, hippocampus, and the basal ganglia. ${ }^{4,65-67}$

\section{BILIRUBIN AND AUTISM SPECTRUM DISORDERS}

In a systematic review ${ }^{68}$ including 13 observational studies, high TSB was associated with autism spectrum disorders (ASD) in term infants (OR 1.43, 95\% Cl 1.22-1.67, random effect model) but not in preterm infants. The limitations in this meta-analysis were selection bias and difficulty in controlling for confounding factors, and therefore, a causal relationship between hyperbilirubinemia and ASD cannot be established. Cerebellar hypoplasia with significantly decreased number of Purkinje cells is the most consistent pathological finding in infants with ASD. ${ }^{69}$ Changes in auditory brain stem-evoked responses are abnormal in children with hyperbilirubinemia and ASD. The lack of association between ASD and hyperbilirubinemia in preterm infants may be due to the fact that free UCB can be significantly higher despite low TSB levels. ${ }^{68}$ Future studies should include measuring free UCB and correlating with ASD in preterm infants.

\section{BILIRUBIN AND ADULT NEUROBEHAVIORAL DISORDERS}

In a 30-year prospective follow-up study on a birth cohort of term and normal birth weight neonates, infants with hyperbilirubinemia 
(>340 $\mu \mathrm{mol} / \mathrm{L}$ ) or needed ET during the neonatal period were more likely to have neurobehavioral symptoms at 9 years of age (OR 4.68) compared to healthy controls. ${ }^{70}$ Forty-five percent of these subjects continued to exhibit cognitive abnormalities in childhood and poor academic achievement, and the ability to finish secondary and tertiary education. Furthermore, $23 \%$ of the affected subjects with a history of hyperbilirubinemia reported cognitive problems at 30 years of age. These findings are suggestive of impairment of the fronto-striatal system. The authors concluded that neonatal hyperbilirubinemia is a recognizable cause of adult age neurobehavioral disorders, severe enough to have negative occupational and quality of life effects. ${ }^{70}$

\section{CLINICAL FEATURES OF BILIRUBIN NEUROTOXICITY}

Acute bilirubin encephalopathy

Acute bilirubin encephalopathy (ABE) may include progressive changes in the infant's mental status, muscle tone, and cry with varying degrees of drowsiness, poor feeding, hypotonia, and alternating tone followed by increasing hypertonia, especially of the extensor muscles, retrocollis, and opisthotonus. ${ }^{13,32}$ Mortality (7-10\%) during the acute stages of $A B E$ is due to respiratory failure and progressive coma or intractable seizures. Rate of progression of clinical signs depends on the rate of bilirubin rise, duration of hyperbilirubinemia, host susceptibility, and presence of comorbidities. ${ }^{10}$ In general, overt neurological abnormalities are uncommon in preterm infants although apnea and bradycardia are increased in preterm infants 25-32 weeks of gestation compared to infants without hyperbilirubinemia. ${ }^{11,71,72}$

In the early phase of $A B E$, infants become lethargic, hypotonic, and suck poorly. The intermediate phase is characterized by moderate stupor, irritability, and hypertonia. The infants may develop fever and high-pitched cry, which may alternate with drowsiness and hypotonia. Hypertonia is manifested by backward arching of the neck (retrocollis) and trunk (opisthotonus). There is anecdotal evidence that ET at this stage in some cases might reverse the central nervous system changes. The advanced phase (irreversible) is characterized by pronounced retrocollis, opisthotonus, shrill cry, poor feeding, apnea, fever, deep stupor to coma, seizures, and death. ${ }^{9,38,53}$ Auditory neuropathy spectrum disorders are a common manifestation in BIND in late preterm and term neonates with severe jaundice. ${ }^{54}$

\section{Kernicterus}

Kernicterus is a chronic disabling neurological disorder classically characterized by the following tetrad, choreoathetoid cerebral palsy, high-frequency sensorineural hearing loss, palsy of vertical gaze, and dental enamel hypoplasia. ${ }^{9,38,53}$ Subtypes have recently been reported and include classical, auditory predominant, and motor predominant presentations. ${ }^{53}$ Although cognitive function in affected children was found to be within normal range, ${ }^{73}$ cognitive developmental delays were found in other studies. ${ }^{74}$ Marked intellectual deficits occur only in the minority of infants. ${ }^{75}$ Unfortunately, most patients have been mistaken to have mental retardation because of their contoured countenance and writhing limb movements as well as undetected auditory dysfunction. The abnormal movements may mask spared cognitive function or auditory deficit may disrupt language development. However, some studies reported that intellect is relatively spared in infants with kernicterus. $^{76}$

The most disabling neurological findings in kernicterus are observed in infants with auditory and motor subtypes. The pattern of involvement may relate to factors such as gestational age and amount and duration of hyperbilirubinemia. ${ }^{.77}$ The auditory sequelae are almost exclusively auditory neuropathy/auditory dyssynchrony (AN/AD) of varying degrees ranging from central auditory processing difficulties with normal hearing to severe AN/AD with absent auditory brain stem responses, normal cochlear microphonic responses with or without accompanying severe-to- profound hearing loss, and deafness. AN/AD is a rare cause of hearing loss and there is good evidence that this is caused by excessive hyperbilirubinemia. ${ }^{78}$

The motor subtype principally involves abnormalities of tone. Hypertonia can be severe resulting in twisted dystonic posturing, episodic sustained muscle spasms, and virtual lack of voluntary movement. Athetosis, a likely manifestation of dystonia is a classical finding with writhing movements that blends continuously into a flowing stream of movement. Paroxysmal extrapyramidal movement disorders in patients with classical kernicterus are occasionally misdiagnosed as seizures and electroencephalogram (EEG) especially, video EEG can distinguish these spells. ${ }^{28}$ Subtle kernicterus refers to individuals with subtle neurodevelopmental disabilities without the classical findings of kernicterus. These may include disturbances in sensory and sensorimotor integration, central auditory processing, coordination, and muscle tone. $^{38}$

\section{Neuroimaging}

The hallmark of magnetic resonance imaging finding in kernicterus is chronic bilateral symmetrically increased T2 signal (or T2 FLAIR signal) in the globus pallidus and subthalamic nucleus. Additionally, increased T2 signal may also be seen in the substantia nigra or in the dentate nucleus of the cerebellum; however, a normal MRI does not exclude the diagnosis. ${ }^{79,80}$ Other advanced neuroimaging techniques include diffusion-weighted imaging, and magnetic resonance spectroscopy (MRS).

\section{MAJOR CAUSES OF HYPERBILIRUBINEMIA}

Hyperbilirubinemia is the result of an imbalance between bilirubin production and elimination. ${ }^{13,21}$ After birth, all neonates have a relatively impaired hepatic conjugation of bilirubin resulting in hyperbilirubinemia that resolves when the conjugating system and other uptake and transport mechanisms responsible for bilirubin elimination mature during the first week of life. Serial TSB measurements can be used to screen for pathologic phenotypes based on hour-specific nomograms to assign different risk zones for developing severe hyperbilirubinemia and thus alert the care providers to initiate treatment to avoid bilirubin neurotoxicity. ${ }^{14}$ The risk zones also reflect relative differences in bilirubin production, the most dramatic of which is hemolytic disease of a newborn. ${ }^{14}$

The major risk factor and serious threat to imbalance are hemolytic conditions and inherited defects in conjugation. Epidemiologic data suggest that there might be an increased risk of neurotoxicity caused by bilirubin in the context of hemolytic disease $^{11,12,21}$ and current recommendations reflect a concern for earlier intervention with phototherapy or exchange transfusions in the presence of hemolysis. ${ }^{14,15}$ Causes of hemolysis can be isoimmune, such as Rh, ABO, and minor blood group incompatibilities, RBC structural changes, like, in hereditary spherocytosis, RBC enzyme disorders, like, G6PD deficiency and pyruvate kinase deficiency, unstable hemoglobin, and micro angiopathic hemolytic disorders. ${ }^{81}$ It is critical to determine blood type and Rh factor at birth and perform direct Coombs test in infants with earlyonset jaundice. However, there is a high percentage of neonates $(\sim 55 \%)$ in whom the risk factors are not obvious. ${ }^{27}$ Other causes of hyperbilirubinemia are decreased clearance of bilirubin, such as in Criggler Najjar syndrome types I and II, Gilbert syndrome, prematurity, and endocrine causes such as, hypothyroidism and hypopituitarism. ${ }^{14,20}$

In full-term infants with severe hyperbilirubinemia secondary to hemolysis, a clear correlation can be discerned between the occurrence of kernicterus and maximal level of bilirubin. A maximum bilirubin level of $30-40 \mathrm{mg} / \mathrm{dL}$ in newborns with hemolytic disease was associated with kernicterus in $73 \%$ of 
cases. ${ }^{27,82}$ Compared to infants without hemolysis, several studies have demonstrated that infants with hemolysis have a higher risk of developing BIND. ${ }^{4,10,83-85}$ An increase in bilirubin production caused by hemolysis, overwhelms bilirubin binding to albumin, ${ }^{86}$ increasing the probability of bilirubin moving into the brain and leading to permanent neuronal injury. ${ }^{87}$ In a recent study, CP consistent with kernicterus occurred in infants with TSB levels more than $5.0 \mathrm{mg} / \mathrm{dL}$ above the exchange transfusion thresholds and with at least two risk factors for neurotoxicity, such as prematurity, G6PD deficiency, or hypoxic-ischemic encephalopathy. ${ }^{88}$

The relationship between neonatal bilirubin values and neurological outcome in preterm infants is different compared to full-term infants. Kernicterus has been reported in preterm and extremely low- birth-weight infants at lower levels of TSB ${ }^{59,89}$ Studies of bilirubin-induced auditory disturbance in preterm infants (28-32 weeks of gestation) demonstrate neurological dysfunction at lower serum TB levels. ${ }^{72,89}$ These infants often have risk factors such as acidosis, sepsis, asphyxia, birth trauma, and hypoglycemia that could predispose these infants to kernicterus at bilirubin levels below phototherapy or exchange transfusion levels. ${ }^{4}$

\section{PREVENTION OF SEVERE HYPERBILIRUBINEMIA}

Universal bilirubin screening

Most cases of neonatal jaundice are benign, but because of the potential toxicity of bilirubin to the developing brain, infants must be monitored to identify those who might develop severe hyperbilirubinemia and in rare cases $A B E$ or kernicterus. Infants with kernicterus from the pilot registry had pre-discharge TSB above the $75 \%$ percentile for age in hours. ${ }^{4,9} \mathrm{TCB}$ has been used in birthing centers and outpatient settings for universal screening and follow-up of bilirubin levels in newborn infants ${ }^{14,15}$ TCB for universal screening of bilirubin is easy to use, non-invasive, painfree with immediate results, and superior to visual estimation of jaundice. $^{90}$ Its use can also decrease the number of TSB determinations and expense. However, any value that approaches treatment threshold should be confirmed with a TSB. TCB screening when initiated within the first $6 \mathrm{~h}$ after delivery has good predictive value regarding subsequent development of hyperbilirubinemia. ${ }^{90}$ Predischarge TcB/TSB adjusted for postnatal age in infants $>35$ weeks of gestation combined with specific risk factors (especially GA, bruising, family history, and rapid rise in TSB) ${ }^{91}$ best predicts TSB $>25 \mathrm{mg} / \mathrm{dL}$ and need for phototherapy. ${ }^{90,92}$ However, screening is sensitive but not specific for predicting exchange transfusion needs. ${ }^{93}$ TcB has been validated in several studies in preterm infants $>30$ weeks of gestations. At present, there are no studies validating the reliability of $T C B$ in preterm infants $<29$ weeks of gestation.

The long-term benefits of universal screening for hyperbilirubinemia have not been demonstrated for TCB screening; ${ }^{94}$ however, occurrence of TSB values of $25-29 \mathrm{mg} / \mathrm{dL}$ decreased from 45 to 27 per 100,000 and values $>30 \mathrm{mg} / \mathrm{dL}$ decreased from 9 to 3 per 100,000 after implementing TCB screening protocol. ${ }^{90,95}$ In a study from Canada, 55\% reduction in the incidence of TSB $>20$ $\mathrm{mg} / \mathrm{dL}$ and decrease in TSB draws with use of phototherapy, and age at readmission for phototherapy after implementation of a screening protocol were reported. ${ }^{90,96}$ In California, analysis of a statewide database found a documented decrease in the incidence of extreme hyperbilirubinemia and rescue exchange transfusions after implementing routine predischarge screening. ${ }^{97}$

Using the nomogram, a predischarge TcB/TSB value above the 75 \%ile for age in hours in healthy babies is categorized as having a clinically significant bilirubin load attributable to increased hemolysis or unrecognized impaired bilirubin clearance. ${ }^{98-100} \mathrm{~A}$ predischarge TcB/TSB in the 75-95th \%ile has 54\% probability of developing hyperbilirubinemia and a predischarge TcB/TSB > 95\% ile approaches $100 \%$ probability of hyperbilirubinemia in infants $<38$ weeks of gestation. ${ }^{14,15,92,101}$ There are no clinical tests or point-of-care devices currently available that can reliably detect ongoing hemolysis. During the breakdown of heme, equimolar amounts of carbon monoxide (CO) and bilirubin are produced, and measurement of end-tidal CO (ETCO) or carboxy hemoglobin $(\mathrm{COHb})$ can be used as indices of hemolysis. However, there is currently no commercial device available to measure bedside ETCO or $\mathrm{COHb}^{14,15}$

\section{Management of hyperbilirubinemia}

A safe and effective way to control production of the bilirubin pigment would be a rational and effective strategy of preventing kernicterus. Apart from TSB measurements, serum albumin level should be measured and the bilirubin-to-albumin ratio $(B / A)$ should be used in conjunction with the TSB level and other risk factors to determine the need for phototherapy or exchange transfusion. ${ }^{14,15,102}$

\section{Phototherapy}

Phototherapy is the treatment of choice to reduce the severity of unconjugated hyperbilirubinemia, regardless of etiology and has been used to treat newborns with jaundice for over half a century. ${ }^{103}$ Bilirubin upon exposure to light undergoes geometric isomerization, structural isomerization (lumirubin), and photooxidation to biliverdin, or mono- and di-pyroles. These different products are eliminated in the bile and urine. Formation of lumirubin is the most important effect of phototherapy. Some studies have shown that the conversion of bilirubin to more polar photo-isomers has a direct neuroprotective effect as they are less prone to cross the blood-brain barrier. ${ }^{104}$ Efficacy of phototherapy is related to intensity of light $\left(\mu \mathrm{W} / \mathrm{cm}^{2} / \mathrm{nm}\right)$, body surface area, and the distance between light source and infant's skin. Conventional phototherapy refers to irradiance around $15 \mu \mathrm{W} / \mathrm{cm}^{2} / \mathrm{nm}$ and intensive phototherapy refers to irradiance around $30 \mu \mathrm{W} / \mathrm{cm}^{2} / \mathrm{nm}^{14}$ Phototherapy was $85 \%$ effective in preventing TSB $\geq 25 \mathrm{mg} / \mathrm{dL}$ and the efficacy of phototherapy in reducing excessive UCB has significantly decreased the need for ET. ${ }^{101,105}$ With intensive phototherapy, a $2 \mathrm{mg} / \mathrm{dL}$ decrease can be achieved within 4-6 h. ${ }^{106}$ Intensive phototherapy is recommended in infants with risk factors, like, sepsis, lethargy, serum albumin $<3 \mathrm{~g} / \mathrm{dL}$, G6PD deficiency, acidosis, iso-immune hemolytic disease, and temperature instability. ${ }^{14,15}$ Phototherapy use has been presumed to be safe; however, recently, there have been reports of a slightly increased risk of childhood cancer with the use of phototherapy, ${ }^{107}$ especially in infants with Down syndrome although the absolute risk is small. ${ }^{108}$ Concern for a possible risk of photo-oxidative injury has been raised in ELBW infants ${ }^{103}$ and increase in mortality among infants with birth weights between 501 and 750 g. $^{109}$

\section{Exchange transfusion}

Exchange transfusion (ET) has been universally established as an effective therapy for severe neonatal hyperbilirubinemia in infants not responding to phototherapy. ET is a rescue procedure for severe hyperbilirubinemia in underdeveloped countries compared to developed countries because of the absence of communitybased use of phototherapy, suboptimal or ineffective phototherapy devices, delayed recognition of excessive bilirubin levels, late referrals, and higher prevalence of G6PD deficiency. ${ }^{110}$ In the United States, it is estimated that the use of ET for serum TB $>30 \mathrm{mg} / \mathrm{dL}$ is approximately 3 per 100,000 live births. The current indications for ET are outlined for infants 35 weeks or greater. ${ }^{14,15}$ For preterm and low-birth-weight infants $<35$ weeks of gestation, guidelines for using phototherapy and ET have been published based on gestational age and TSB values. ${ }^{102,111}$ However, there are no studies validating these recommendations. Other forms of therapy for hyperbilirubinemia secondary to 


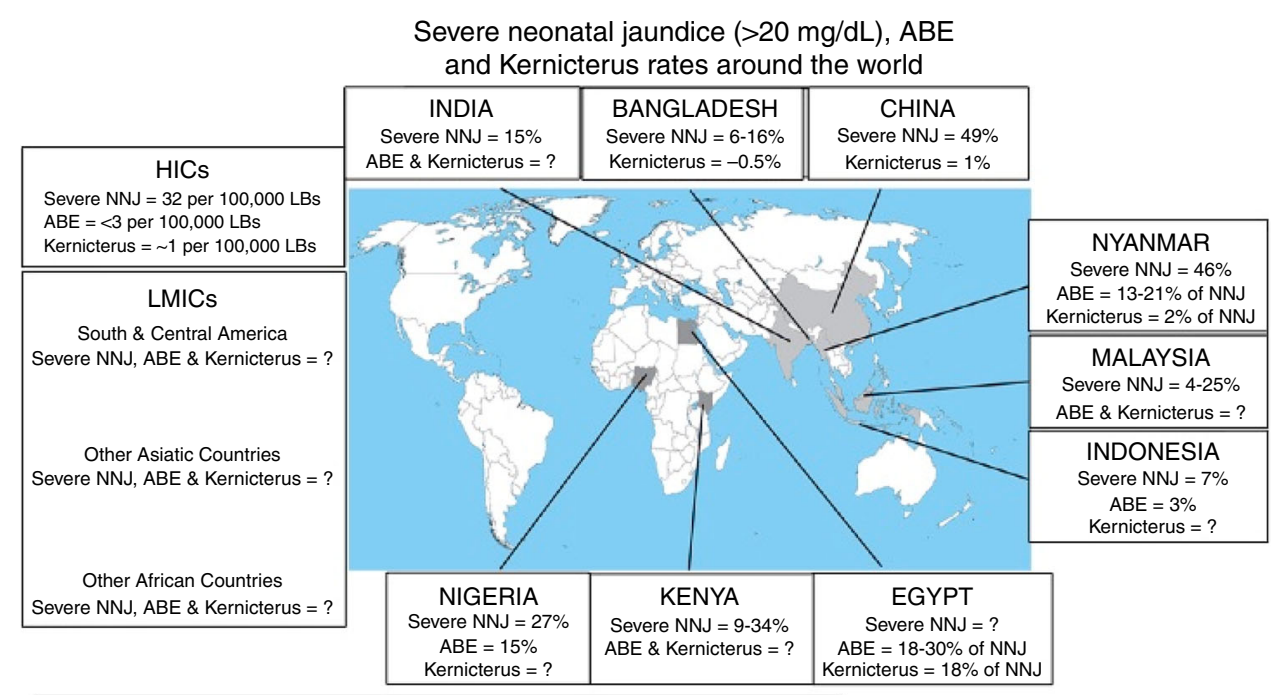

Data reported refer to hospital statistics; No national Data available; NNJ = Neonatal Jaundice; LBs = live births; $\mathrm{ABE}=$ acute bilirubin encephalopathy

Greco $C$ et al. Neonatology 2016;110:172-180

Fig. 3 Rates of severe hyperbilirubinemia, $A B E$ and kernicterus around the world

iso-immune hemolysis is the use of intravenous immune globulin to block immunoglobulin constant fragment receptors and inhibit hemolysis. ${ }^{14}$ Another approach to prevent hyperbilirubinemia is the pharmacologic approach with the use of Tn-mesoporphyrin, to block heme oxygenase, the rate-limiting enzyme in bilirubin production. ${ }^{14,21,27}$

The re-emergence of kernicterus in the United States is likely due to breakdown in health systems for newborn jaundice management. The most prevalent potentially correctable root cause is early discharge combined with failure of timely follow up of late preterm and term neonates. ${ }^{9}$ Other problems include failure to recognize the presence of risk factors such as exclusive breastfeeding, late preterm infants, presence of iso-immune or other hemolytic diseases, previous sibling with jaundice, cephalohematoma or extensive bruising, and east Asian race. ${ }^{13,112}$ Delayed cord clamping with higher Hgb levels has also been a factor for increased need for phototherapy. ${ }^{113}$

Severe neonatal jaundice is associated with a significant health burden especially in LMIC. True incidence of BIND and kernicterus is underreported due to lack of regional or national databases in many countries ${ }^{8,22}$ (Fig. 3). Regardless of the cause of jaundice, the potential risk for unrecognized, unmonitored, and untreated severe hyperbilirubinemia is a major risk factor for neurotoxicity in preterm and term neonates. As kernicterus is preventable, but not treatable, our focus needs to be rooted in the prevention strategies. There is an urgent need to educate clinicians and families about the warning signs of bilirubin neurotoxicity because early and intermediate stages of $A B E$ may be reversible with prompt and effective bilirubin reduction strategies. ${ }^{114}$ In addition, introduction of inexpensive and simple-to-use devices for measuring bilirubin and treating neonatal jaundice ${ }^{115}$ is essential for the prevention of bilirubin-induced neurotoxicity globally.

\section{ADDITIONAL INFORMATION}

Competing interests: The authors declare no competing interests.

Publisher's note: Springer Nature remains neutral with regard to jurisdictional claims in published maps and institutional affiliations.

\section{REFERENCES}

1. Bhutani, V. K., Johnson, L. \& Sivieri, E. M. Predictive ability of a pre-discharge hour-specific serum bilirubin for subsequent significant hyperbilirubinemia in healthy term and near-term newborns. Pediatrics 103, 6-14 (1999).

2. Newman, T. B. et al. Jaundice and the infant study team. Outcomes among newborns with total serum bilirubin levels of $25 \mathrm{mg} / \mathrm{dL}$ or more. N. Engl. J. Med. 354, 1889-1900 (2006).

3. Bhutani, V. K. \& Johnson, L. H. Urgent clinical need for accurate and precise bilirubin measurements in the United States to prevent kernicterus. Clin. Chem. 50, 477-480 (2004).

4. Shapiro, S. Bilirubin toxicity in the developing nervous system. Pediatr. Neurol. 29, 410-421 (2003).

5. Sgro, M., Campbell, D. M., Kandasamy, S. \& Shah, V. Incidence of chronic bilirubin encephalopathy in Canada 2007-2008. Pediatrics 130, e886-e890 (2012).

6. Manning, D., Todd, P., Maxwell, M. \& Jane, P. M. Prospective surveillance study of severe hyperbilirubinemia in the newborn in the UK and Ireland. Arch. Dis. Child. Fetal Neonatal Ed. 92, F342-F346 (2007).

7. Ebbesen, F., Bjerre, J. V. \& Vandborg, P. K. Relation between serum bilirubin levels $\geq 450 \mathrm{Imol} / \mathrm{L}$ and bilirubin encephalopathy; a Danish population-based study. Acta Pædiatr 101, 384-389 (2012).

8. Greco, C. et al. Neonatal jaundice in low- and middle-income countries: lessons and future directions from the 2015 Don Ostrow Trieste Yellow Retreat. Neonatology 110, 172-180 (2016).

9. Johnson, L., Bhutani, V. K., Karo, K., Sivieri, E. M. \& Shapiro, S. M. Clinical report from the Pilot USA Registry. J. Perinatol. 29, S25-S45 (2009).

10. Johnson, L. \& Bhutani, V. K. The clinical syndrome of bilirubin induced neurologic dysfunction. Semin. Perinatol. 35, 101-113 (2011).

11. Rose, J. \& Vassar, R. Movement disorders due to bilirubin toxicity. Semin. Fetal Neonat. Med. 20, 20-25 (2015).

12. Wong, R. J. \& Stevenson, D. K. Neonatal hemolysis and risk of bilirubin induced neurologic dysfunction. Semin. Fetal Neonat. Med. 20, 26-30 (2015).

13. Kaplan, M., Bromiker, R. \& Hammerman, C. Hyperbilirubinemia, hemolysis and increased bilirubin toxicity. Semin. Perinatol. 38, 429-437 (2014).

14. American Academy of Pediatrics Subcommittee on Hyperbilirubinemia.. Management of hyperbilirubinemia in the newborn infant 35 or more weeks of gestation. Pediatrics 114, 297-316 (2004).

15. Maisels, J. et al. Hyperbilirubinemia in the newborn infant $\geq 35$ weeks gestation: An update with clarifications. Pediatrics 124, 1193-1198 (2009).

16. Bhutani, V. K., Vilms, R. J. \& Hammerman-Johnson, L. Universal bilirubin screening for severe neonatal hyperbilirubinemia. J. Perinatol. 30, S6-S15 (2010).

17. Kuzniewicz, M. W., Escobar, G. J. \& Newman, T. B. Impact of universal bilirubin screening on severe hyperbilirubinemia and phototherapy use. Pediatrics 124, 1031-1039 (2009). 
18. Trikalinos, T. A., Ching, M., Lau, J. \& Ip, S. Systematic review of screening for bilirubin encephalopathy in neonates. Pediatrics 124, 1162-1171 (2009).

19. Kaplan, M., Bromiker, R. \& Hammerman, C. Severe neonatal hyperbilirubinemia and kernicterus: are these still problems in the third millennium? Neonatology 100, 354-362 (2011).

20. Maisels, M. J. Neonatal jaundice. Pediatr. Rev. 27, 443-454 (2006).

21. Stevenson, D. K., Vreman, H. J. \& Wong, R. J. Bilirubin production and the risk of neurotoxicity. Semin. Perinatol. 35, 212-126 (2011).

22. Slusher, T. M. et al. Burden of severe neonatal jaundice: a systematic review and meta-analysis. BMJ Paediatr. Open 1, e000105 (2017).

23. De Carvalho, M., Robertson, S. \& Klaus, M. Fecal bilirubin excretion and serum bilirubin concentrations in breastfed and bottle-fed infants. J. Pediatr. 107, 786-790 (1985).

24. Gourley, G. R., Kreamer, B. \& Arend, R. The effect of diet on feces and jaundice during the first 3 weeks of life. Gastroenterology 103, 660-667 (1992).

25. Falcao, A. S. et al. Apoptosis and impairment of neurite network by short exposure of immature rat cortical neurons to unconjugated bilirubin increase with cell differentiation and are additionally enhanced by an inflammatory stimulus. J. Neurosci. Res. 85, 1229-1239 (2007).

26. Vaz, A. R. et al. Bilirubin selectively inhibits cytochrome c oxidase activity and induces apoptosis in immature cortical neurons: assessment of the protective effects of glycoursodeoxycholic acid.. J. Neurochem. 112, 55-65 (2010).

27. Bertini, G., Dani, C., Pezzati, M. \& Rubaltelli, F. Prevention of bilirubin encephalopathy. Biol. Neonate. 79, 219-223 (2001).

28. Volpe, J. J. Bilirubin and Brain Injury Neurology of the Newborn. 5th edn, 619-665 (W. B. Saunders, Philadelphia, 2008).

29. Brites, D. \& Fernandes, A. Bilirubin induced neural impairment: a special focus on myelination, age related windows of susceptibility and associated co-morbidities. Semin Fetal Neonat. Medicine 20, 14-19 (2015).

30. Brodersen, R. \& Stern, L. Deposition of bilirubinic acid in the central nervous system--a hypothesis for the development of kernicterus. Acta Paediatr. Scand. 79, 12-19 (1990).

31. Brites, D. Bilirubin injury to neurons and glial cells: new players, novel targets and newer insights. Semin. Perinatol. 35, 114-120 (2011).

32. Silva, R. F., Rodrigues, C. M. \& Brites, D. Rat cultured neuronal and glial cells respond differently to toxicity of unconjugated bilirubin. Pediatr. Res. 51, 535-541 (2002).

33. Usman, F., Diala, U. M., Shapiro, S. M., Le Pichon, J. B. \& Slusher, T. M. Acute bilirubin encephalopathy and its progression to kernicterus: current perspectives. Res. Rep. Neonatol. 8, 33-44 (2018).

34. Ostrow, J. D., Pascolo, L. \& Tiribelli, C. Reassessment of the unbound concentrations of unconjugated bilirubin in relation to neurotoxicity in vitro. Pediatr. Res. 54, 98-104 (2003)

35. Falcao, A. S., Fernandes, A., Brito, M. A., Silva, R. F. \& Brites, D. Bilirubin induced inflammatory response, glutamate release and cell death in rat cortical astrocytes are enhanced in younger cells. Neurobiol. Dis. 20, 199-206 (2005).

36. Fernandes, A., Silva, R. F., Falcao, A. S., Brito, M. A. \& Brites, D. Cytokine production, glutamate release and cell death in rat cultured astrocytes treated with unconjugated bilirubin and LPS. J. Neuroimmunol. 153, 64-75 (2004).

37. Ostrow, J. D., Pascolo, L., Brites, D. \& Trielli, C. Molecular basis of bilirubin induced neurotoxicity. Trends Mol. Med. 10, 65-70 (2004).

38. Shapiro, S. Definition of clinical spectrum of kernicterus and bilirubin induced neurologic dysfunction. J. Perinatol. 25, 54-59 (2005).

39. Watchko, J. F. Kernicterus and the molecular mechanisms of bilirubin induced CNS injury in the newborns. Neuromolecular. Med. 8, 513-529 (2006).

40. Brito, M. A., Brites, D. \& Butterfield, D. A. A link between hyperbilirubinemia, oxidative stress and injury to neocortical synaptosomes. Brain Res. 1026, 33-43 (2004).

41. Brites, D. The evolving landscape of neurotoxicity by unconjugated bilirubin: role in glial cells and inflammation. Front. Pharmacol. 3, 88 (2012).

42. Hansen, T. W., Mathiesen, S. B. \& Walaas, S. I. Bilirubin has widespread inhibitory effects on protein phosphorylation. Pediatr. Res. 39, 1072-1077 (1996).

43. Amin, S. B. \& Lamola, A. A. Newborn jaundice technologies: unbound bilirubin and bilirubin binding capacity in neonates. Semin. Perinatol. 35, 134-140 (2011).

44. Ahlfors, C. E., Amin, S. B. \& Parker, A. E. Unbound bilirubin predicts abnorma automated auditory brainstem response in a diverse newborn population. J. Perinatol. 29, 305-309 (2009).

45. Nakamura, H., Yonetani, M., Uetani, Y., Funato, M. \& Lee, Y. Determination of serum unbound bilirubin for prediction of kernicterus in low birth weight infants. Acta Paediatr. Jpn. 34, 642-647 (1992).

46. Cashore, W. J. \& Oh, W. Unbound bilirubin and kernicterus in low birth weight infants. Pediatrics 69, 481-485 (1982).

47. Amin, S. B. et al. Chronic auditory toxicity in late preterm and term infants with significant hyperbilirubinemia. Pediatrics 140, e20164009 (2017).
48. Amin, S. B., Wang, H., Laroia, N. \& Orlando, M. Unbound bilirubin and auditory neuropathy spectrum disorder in late preterm and term infants with severe jaundice. J. Pediatr. 173, 84-89 (2016).

49. Haymaker, W., Margles, C. \& Penstchew, A. in Kernicterus and Importance in Cerebral Palsy (ed. Swinyard, C. A.) pp. 21-229 (Charles C. Thomas, Springfield, 1961).

50. Ahdab-Barmada, M. \& Moosy, J. The neuropathology of kernicterus in the premature neonate. Diagnostic problems. J. Neuropathol. Exp. Neurol. 43, 45-56 (1984).

51. Claireaux, A. E. in Pathology of Human Kernicterus (University of Toronto Press, Montreal, 1961).

52. Watchko, J. F. \& Tiribelli, C. Bilirubin induced neurologic damage-mechanisms and management approaches. New Eng. J. Med. 369, 2021-2030 (2013).

53. Shapiro, S. Chronic bilirubin encephalopathy: diagnosis and outcome. Semin Fetal Neonatal Med 15, 157-163 (2010).

54. Lunsing, R. J. Subtle bilirubin induced neurodevelopmental dysfunction (BIND) in the term and late preterm infant. Does it exist? Semin. Perinatol. 38, 456-471 (2014).

55. Bortolussi, G. et al. Impairment of enzymatic antioxidant defenses is associated with bilirubin-induced neuronal cell death in the cerebellum of Ugt1 KO mice. Cell Death Dis. 6, e1739 (2015).

56. Rhine, W. D., Schmitter, S. P., Yu, A. C., Eng, L. F. \& Stevenson, D. K. Bilirubin toxicity and differentiation of cultured astrocytes. J. Perinatol. 19, 206-211 (1999).

57. Fernandes, A. et al. Bilirubin as a determinant for altered neurogenesis, neuritogenesis, and synaptogenesis. Dev. Neurobiol. 69, 568-62 (2009).

58. Rodrigues, C. M. P., Sola, S., Silva, R. F. M. \& Brites, D. Aging confers different sensitivity to the neurotoxic properties of unconjugated bilirubin. Pediatr. Res. 51, 112-118 (2002)

59. Watchko, J. F. \& Maisels, M. J. The enigma of low bilirubin kernicterus in premature infants. Why does it still occur and is it preventable? Semin. Perinatol. 38 397 (2014).

60. Morioka, I., Iwatani, S., Koda, T., lijima, K. \& Nakamura, H. Disorders of bilirubin binding to albumin and bilirubin induced neurologic function. Semin. Fetal Neonat. Med. 20, 31-36 (2015).

61. Amin, S. B. \& Wang, H. Bilirubin albumin binding and unbound unconjugated hyperbilirubinemia in premature infants. J. Pediatr. 192, 47-52 (2018).

62. Hansen, T. W. R., Maynard, E. C., Cashore, W. J. \& Oh, W. Endotoxemia and brain bilirubin in the rat. Biol. Neonate. 63, 171-176 (1993).

63. Petty, M. A. \& Lo, E. H. Junctional complexes of the blood brain barrier: permeability change in neuroinflammation. Prog. Neurobiol. 68, 311-323 (2002).

64. Graziani, L. J. et al. Neurodevelopment of preterm infants: neonatal neurosonographic and serum bilirubin studies. Pediatrics 89, 229-234 (1992).

65. Johnson, L., Sarmiento, F., Blanc, W. A. \& Day, R. Kernicterus in rats with an inherited deficiency of glucuronyl transferase. Am. J. Dis. Child. 97, 591-608 (1959).

66. Schutta, H. S. \& Johnson, L. Bilirubin encephalopathy in the Gunn rat. A fine structure of the cerebellar cortex. J. Neuropathol. Exp. Neurol. 26, 377-396 (1967).

67. Schutta, H. S. \& Johnson, L. Clinical signs and morphologic abnormalities in Gunn rats treated with Sulfadimethoxine. J. Pediatr. 75, 1070-1079 (1969).

68. Amin, S. B., Smith, T. \& Wang, H. Is neonatal jaundice associated with autism spectrum disorders: a systematic review. J. Autism Dev. Diord 41, 1455-1463 (2011).

69. Bauman, M. L. \& Kemper, T. L. Neuroanatomic observations of the brain in autism: a review and future directions. Int. J. Dev. Neurosci. 23, 183-187 (2005).

70. Hokkanen, L., Launes, J. \& Michelsson, K. Adult neurobehavioral outcome of hyperbilirubinemia in full term neonates-a 30 year prospective follow-up study. PeerJ 2, e294 (2014).

71. Amin, S. B. Clinical assessment of bilirubin induced neurotoxicity in premature infants. Semin. Perinatol. 28, 340-347 (2004).

72. Amin, S. B., Charafeddine, L. \& Guillet, R. Transient bilirubin encephalopathy and apnea of prematurity in $28-32$ weeks gestational age infants. J. Perinatol. 25, 386-390 (2005).

73. Rubin, R. A., Balow, B. \& Fisch, R. O. Neonatal serum bilirubin levels related to cognitive development at ages 4 through 7. J. Pediatr. 94, 601-604 (1979).

74. Seidman, D. S., et al. Neonatal hyperbilirubinemia and physical and cognitive performance at 17 years of age. Pediatrics 88, 828-833 (1991).

75. Byers, R. K., Paine, R. S. \& Crothers, B. Extrapyramidal cerebral palsy with hearing loss following erythroblastosis. Pediatrics 15, 248-254 (1955).

76. Ip, S. et al. An evidenced based review of important issues concerning hyperbilirubinemia. Pediatrics 114, e130-e153 (2004).

77. Shapiro, S. M. \& Daymont, M. J. Patterns of kernicterus related to neonatal hyperbilirubinemia and gestational age. Pediatr. Res 53(4 Part2), 398A-399A (2003). 
High unbound bilirubin for age: a neurotoxin with major effects on the... R Cayabyab and R Ramanathan

78. Saluja, S., Agarwal, A., Kier, N. \& Amin, S. Auditory neuropathy spectrum disorder in late and preterm infants with severe jaundice. Int. J. Pediatr. Otorhinolaryngol. 74, 1292-1297 (2010).

79. Wisnowski, J. L., Panigrahy, A., Painter, M. J. \& Watchko, J. Magnetic resonance imaging of bilirubin encephalopathy: current limitations and promise. Semin. Perinatol. 38, 422-428 (2014).

80. Yilmaz, Y. et al. Magnetic resonance imaging findings in patients with severe neonatal indirect hyperbilirubinemia. J. Child Neurol. 16, 452-455 (2001).

81. Christensen, R. D. \& Yaish, H. M. Hemolytic disorders causing severe neonatal hyperbilirubinemia. Clin. Perinatol. 42, 515-527 (2015).

82. Mollison, P. L. \& Cutbush, M. A method of measuring the severity of a series of cases of hemolytic disease of the newborn. Blood 6, 777-788 (1951).

83. Newman, T. B. \& Maisels, M. J. Response to commentaries re: evaluation and treatment of jaundice in term newborn: A kinder, gentler approach. Pediatrics 89, 831-833 (1992).

84. Watchko, J. F. \& Oski, F. A. Bilirubin $20 \mathrm{mg} / \mathrm{dL}=$ vigintiphobia. Pediatrics 71, 660-663 (1983).

85. Bhutani, V. K. \& Johnson-Hammerman, L. The clinical syndrome of bilirubin induced neurologic dysfunction. Semin Fetal Neonatal Med. 20, 6-13 (2015).

86. Wennberg, R. P., Ahlfors, C., Bhutani, V. K., Johnson, L. \& Shapiro, S. Toward understanding kernicterus: a challenge to improve the management of jaundiced newborns. Pediatrics 117, 474-485 (2006).

87. Brites, D. et al. Biological risks, for neurological abnormalities associated with hyperbilirubinemia. J. Perinatol. 29(Suppl), S8-S13 (2009).

88. Wu, Y. W. et al. Risk for cerebral palsy in infants with total serum bilirubin levels at or above the exchange transfusion threshold: a population-based study. JAMA Pediatr. 163, 239-246 (2015).

89. Moll, M., Goelz, R., Naegele, T., Wilke, M. \& Poets, C. F. Are recommended phototherapy thresholds safe enough for extremely low birth weight infants (ELBW)? A report on 2 ELBW infants with kernicterus despite only moderate hyperbilirubinemia. Neonatology 99, 90-94 (2011).

90. Engle, W. D., Jackson, G. L. \& Engle, N. G. Transcutaneous bilirubinometry. Semin. Perinatol. 38, 438-451 (2014).

91. Bhutani, V. K. et al. Predischarge screening for severe neonatal hyperbilirubinemia identifies infants who need phototherapy. J. Pediatr. 162, 477-482 (2013).

92. Kuzniewicz, M. W. et al. Risk factors for severe hyperbilirubinemia among infants with borderline bilirubin levels: A nested case-control study. J. Pediatr. 153, 234-240 (2008).

93. Flaherman, V. J., Kuzniewicz, M. W., Escobar, G. J. \& Newman, T. B. Total serum bilirubin exceeding exchange transfusion thresholds in the setting of universal screening. J. Pediatr. 160, 796-800 (2012).

94. US Preventive Services Task Force. Screening of infants for hyperbilirubinemia to prevent chronic bilirubin encephalopathy: US Preventive Task Force Recommendation. Pediatrics 124, (1172-1177 (2009).

95. Mah, M. et al. Reduction of severe hyperbilirubinemia after institution of predischarge bilirubin screening. Pediatrics 125, e1143-e1148 (2010).
96. Wainer, S., Parmar, S. Allegro, D., Rabi, Y. \& Lyon, M. E. Impact of a transcutaneous bilirubinometry program on resource utilization and severe hyperbilirubinemia. Pediatrics 129, 77-86 (2012).

97. Bhutani, V. K. et al. Extreme hyperbilirubinemia and rescue exchange transfusion in California from 2007-2012. J. Perinatol. 36, 853-857 (2016).

98. Bhutani, V. K. \& Johnson, L. H. Jaundice technologies. Prediction of hyperbilirubinemia in term and near term newborns. J. Perinat. 21(Suppl 1), S76-S82 (2001).

99. Dennery, P. A., Seidman, D. \& Stevenson, D. K. Neonatal hyperbilirubinemia. N. Engl. J. Med. 334, 581-590 (2001).

100. Stevenson, D. K. et al. Prediction of hyperbilirubinemia in near-term and term infants. Pediatrics 108, 31-39 (2001).

101. Keren, R. et al. A comparison of alternative risk strategies for predicting significant hyperbilirubinemia in term and near -term infants. Pediatrics 121, e170-e179 (2008).

102. Maisels, M. J. et al. An approach to the management of hyperbilirubinemia in the preterm infant less than 35 weeks of gestation. J. Perinatol. 32, 660-664 (2012).

103. Stevenson, D. K., Wong, R. J., Arnold, C. C., Pedroza, C. \& Tyson, J. E. Phototherapy and the risk of photooxidative injury in extremely low birth weight infants. Clin. Perinatol. 43, 291-295 (2016).

104. Hansen, T. The roles of phototherapy in the crash cart approach to extreme neonatal jaundice. Semin. Perinatol. 35, 171-174 (2011).

105. Wickermasinghe, A. C., Kuzniewicz, M. W., McCullough, C. E. \& Newman, T. H. Efficacy of subthreshold newborn phototherapy during the birth hospitalization in preventing readmission for phototherapy. JAMA Pediatr. 172, 378-385 (2018).

106. Bhutani, V. K. Committee of the Fetus and Newborn. Phototherapy to prevent severe neonatal hyperbilirubinemia in the newborn infants 35 weeks or more of gestation. Pediatrics 128, e1046-e1052 (2011).

107. Wickremasinghe, A. C. et al. Neonatal phototherapy and infantile cancer. Pediatrics 137, e20151353 (2016).

108. Newman, T. B. et al. Retrospective cohort study and childhood cancer in Northern California. Pediatrics 137, e20151354 (2016).

109. Morris, B. H. et al. Aggressive vs. conservative phototherapy for infants with extremely low birth weight. N. Engl. Med 359, 1885-1896 (2008). 18.

110. Olusanya, B. O., Imam, Z. O., Emokpae, A. A. \& Iskander, I. F. Revisiting the criteria for exchange transfusion for severe neonatal hyperbilirubinemia in resourcelimited settings. Neonatology 109, 97-104 (2016).

111. Murki, S. \& Kumar, P. Blood exchange transfusion for infants with severe neonatal hyperbilirubinemia. Semin. Perinatol. 35, 175-184 (2011).

112. Maisels, M. J. Neonatal hyperbilirubinemia and Kernicterus-Not gone but sometimes forgotten. Early Hum. Dev. 85, 727-732 (2009).

113. Nakagawa, M. et al. Correlation between umbilical cord hemoglobin and rate of jaundice requiring phototherapy in healthy newborns. Pediatr. Int. 57, 626-628 (2015).

114. Bhutani, V. K. et al. Kernicterus: epidemiological strategies for its prevention through systems-based approaches. J. Perinatol. 24, 650-662 (2004).

115. Bhutani, V. K., Cline, B. K., Donaldson, K. M. \& Vreman, H. J. The need to implement effective phototherapy in resource constrained settings. Semin. Perinatol. 35, 192-19 (2011). 